\title{
POSITIVE SOLUTIONS OF SINGULAR DIRICHLET PROBLEMS VIA VARIATIONAL METHODS
}

\author{
Juntao Sun And Jifeng Chu
}

\begin{abstract}
In this paper, we establish the existence results for second order singular Dirichlet problems via variational methods. Some recent results are extended and improved. Examples are also given to illustrate the new results.
\end{abstract}

\section{Introduction}

We are devoted to establish the existence results for the following secondorder non-autonomous Dirichlet problems

$$
\left\{\begin{array}{l}
-u^{\prime \prime}=\lambda f(t, u), \quad 0<t<1, \\
u(0)=u(1)=0,
\end{array}\right.
$$

where $\lambda \geq 1$ is a parameter and $f \in \mathbb{C}((0,1) \times(0, \infty),[0, \infty))$ satisfies the following condition

(A) there exist some positive constants $\epsilon, C$ ( $C$ could be dependent of $\epsilon$ ) and $\alpha \in(0,1)$ such that

$$
2 \epsilon \leq f(t, u) \leq C u^{-\alpha}, \quad(t, u) \in(0,1) \times(0, \epsilon) .
$$

Therefore, $f$ may be singular at $u=0$. A typical example is

$$
f(t, u)=u^{-\alpha}+g(t, u)
$$

with $g \in \mathbb{C}((0,1) \times[0, \infty),[0, \infty))$.

Problem (1.1) arises in the transport of coal slurries down conveyor belts [19], boundary layer theory [10] and chemical reactor theory [6, 7]. Due to the

Received June 9, 2011; Revised February 14, 2013.

2010 Mathematics Subject Classification. 34B15.

Key words and phrases. positive solutions, singular Dirichlet problems, variational methods, critical points.

Juntao Sun was supported by the National Natural Science Foundation of China (Grant No. 11201270, No.11271372), Shandong Natural Science Foundation (Grant No. ZR2012AQ010), and Young Teacher Support Program of Shandong University of Technology. Jifeng Chu was supported by the National Natural Science Foundation of China (Grant No. 11171090), the Program for New Century Excellent Talents in University (Grant No. NCET10-0325), China Postdoctoral Science Foundation funded project (Grant 2012T50431). 
important applications, problem (1.1) has received considerable attention during the last few decades. We refer the reader to $[2,3,13,16,20]$. Some classical tools have been used to study (1.1) in literatures, including the method of upper and lower solutions [4, 14], some fixed point theorems [11, 17], a nonlinear Leray-Schauder alternative principle [3, 11] and the variational approach [5]. Among those results, an important existence result was established by Agarwal and O'Regan in [3], in which it was proved that the scalar problem

$$
\left\{\begin{array}{l}
u^{\prime \prime}+q(t) f(t, u)=0, \quad 0<t<1, \\
u(0)=u(1)=0
\end{array}\right.
$$

has at least two positive solutions under some natural conditions, based on the Leray-Schauder alternative principle and a well-known fixed point theorem on cones. In particular, they obtained that the following problem

$$
\left\{\begin{array}{l}
u^{\prime \prime}+\frac{1}{\alpha+1}\left(u^{-\alpha}+u^{\beta}+1\right)=0, \quad 0<t<1 \\
u(0)=u(1)=0, \quad \alpha>0, \quad \beta>1
\end{array}\right.
$$

admits two positive solutions $u_{1}, u_{2} \in \mathbb{C}[0,1] \cap \mathbb{C}^{2}(0,1)$ with $\left\|u_{1}\right\|<1<$ $\left\|u_{2}\right\|$. Recently, Chu and O'Regan [11] extended the above results to the nonautonomous Dirichlet system

$$
\left\{\begin{array}{l}
u^{\prime \prime}+q(t) f(t, u)+e(t)=0, \quad 0<t<1, \\
u(0)=u(1)=0
\end{array}\right.
$$

in which $e$ is allowed to take negative values.

The present work is mainly motivated by [5], in which Agarwal, Perera and O'Regan established the existence results for problem (1.1) with $\lambda=1$ by variational methods. In particular, they proved that problem (1.1) possesses two positive solutions when the nonlinear term satisfies the asymptotically linear and superlinear conditions at infinity, respectively. Inspired by [5], in this paper we prove that problem (1.1) has at least one positive solution and two positive solutions under the different conditions, respectively. Moreover, we also give the existence result of infinitely many positive solutions for singular problem (1.1), in which the symmetric condition on the nonlinear term is not assumed. The proofs are based on variational methods. It is worth noting that there are some works concerning on the results of infinitely many positive solutions for singular problems up to now. For example, Cid et al. [12] obtained the existence of infinitely many solutions for a second-order singular problem with initial value condition. He and Zou [15] studied a singular elliptic equation involving critical exponents in the whole space $\mathbb{R}^{N}$, and obtained the existence of infinitely many solutions. However, their results cannot cover our case and our results are new compared with those in the literature. 


\section{Preliminaries}

Define $f_{\epsilon} \in \mathbb{C}((0,1) \times \mathbb{R},[0, \infty))$ by

$$
f_{\epsilon}(t, u)=f\left(t,\left(u-\varphi_{\epsilon}(t)\right)^{+}+\varphi_{\epsilon}(t)\right),
$$

where $u^{ \pm}=\max \{ \pm u, 0\}$ and $\varphi_{\epsilon}(t)=\epsilon t(1-t)$ is the solution of

$$
\left\{\begin{array}{l}
-u^{\prime \prime}=2 \epsilon, \quad 0<t<1 \\
u(0)=u(1)=0
\end{array}\right.
$$

Consider

$$
\left\{\begin{array}{l}
-u^{\prime \prime}=\lambda f_{\epsilon}(t, u), \quad 0<t<1 \\
u(0)=u(1)=0
\end{array}\right.
$$

By condition (A) and (2.1), one has

$$
2 \epsilon \leq f_{\epsilon}(t, u) \leq C \varphi_{\epsilon}^{-\alpha}, \quad(t, u) \in(0,1) \times(-\infty, \epsilon),
$$

and

$$
f_{\epsilon}(t, u)=f(t, u), \quad(t, u) \in(0,1) \times[\epsilon, \infty) .
$$

An important observation is that if $u$ is a solution of problem (2.3), then $u \geq \varphi_{\epsilon}$, and hence $u$ is also a solution of problem (1.1). In fact, if not, then suppose that

$$
u\left(t_{0}\right)<\varphi_{\epsilon}\left(t_{0}\right) \text { for some } t_{0} \in(0,1) .
$$

According to [1, Lemma 2.8.1], we obtain

$$
t(1-t)\|u\|_{\infty} \leq u(t) \quad t \in[0,1]
$$

where $\|u\|_{\infty}=\max _{t \in[0,1]}|u(t)|$. So (2.6) implies $\|u\|_{\infty}<\epsilon$. But by (2.4),

$$
-u^{\prime \prime} \geq 2 \lambda \epsilon \geq 2 \epsilon=-\varphi_{\epsilon}^{\prime \prime},
$$

so $u \geq \varphi_{\epsilon}$, contradicting (2.6).

Since $\varphi_{\epsilon}^{-\alpha} \in L^{1}(0,1)$, from (2.4) we see that the solutions of problem (2.3) are the critical points of the $C^{1}$ functional

$$
J(u)=\int_{0}^{1}\left(\frac{1}{2}\left|u^{\prime}(t)\right|^{2}-\lambda F_{\epsilon}(t, u)\right) d t, \quad u \in H=H_{0}^{1}(0,1),
$$

and

$$
\left\langle J^{\prime}(u), v\right\rangle=\int_{0}^{1} u^{\prime}(t) v^{\prime}(t) d t-\lambda \int_{0}^{1} f_{\epsilon}(t, u(t)) v(t) d t
$$

for all $v \in H$, where

$$
F_{\epsilon}(t, u)=\int_{\epsilon}^{u} f_{\epsilon}(t, y) d y
$$


and $H_{0}^{1}(0,1)$ is the usual Sobolev space normed by

$$
\|u\|=\left(\int_{0}^{1}\left|u^{\prime}(t)\right|^{2} d t\right)^{\frac{1}{2}} .
$$

Define two functionals $\Phi$ and $\Psi$ as follows

$$
\Phi(u)=\frac{1}{2}\|u\|^{2} \quad \text { and } \quad \Psi(u)=\int_{0}^{1} F_{\epsilon}(t, u) d t
$$

for each $u \in H$. Then by (2.8),

$$
J(u)=\Phi(u)-\lambda \Psi(u) .
$$

Lemma 2.1. Let $u \in H$. Then $\|u\|_{\infty} \leq\|u\|$.

Proof. For any $u \in H$,

$$
|u(t)|=\left|\int_{0}^{t} u^{\prime}(s) d s\right| \leq \int_{0}^{1}\left|u^{\prime}(s)\right| d s \leq\left(\int_{0}^{1}\left|u^{\prime}(s)\right|^{2} d s\right)^{\frac{1}{2}},
$$

which implies the conclusion.

In the next section, we will use the following three theorems in nonlinear functional analysis and critical point theory.

Theorem 2.2 ([21, Theorem 38.A]). Let $X$ be a Banach space. For the functional $\varphi: M \subseteq X \rightarrow[-\infty,+\infty]$ with $M \neq \emptyset, \min _{u \in M} \varphi(u)=\alpha$ has a solution in case the following hold:

(i) $X$ is a real reflexive Banach space;

(ii) $M$ is bounded and weak sequentially closed;

(iii) $\varphi$ is weak sequentially lower semi-continuous on $M$, i.e., by definition, for each sequence $\left\{u_{n}\right\}$ in $M$ such that $u_{n} \rightarrow u$ as $n \rightarrow \infty$, we have $\varphi(u) \leq$ $\liminf _{n \rightarrow \infty} \varphi\left(u_{n}\right)$ holds.

Theorem 2.3 ([18, Theorem 4.10]). Let $X$ be a Banach space and let $\varphi \in$ $C^{1}(X, \mathbb{R})$. Assume that there exist $x_{0}, x_{1} \in X$ and a bounded open neighbourhood $\Omega$ of $x_{0}$ such that $x_{1} \in X \backslash \bar{\Omega}$ and

$$
\max \left\{\varphi\left(x_{0}\right), \varphi\left(x_{1}\right)\right\}<\inf _{x \in \partial \Omega} \varphi(x) .
$$

Let

$$
\Gamma=\left\{h \in C([0,1], X): h(0)=x_{0}, h(1)=x_{1}\right\}
$$

and

$$
c=\inf _{h \in \Gamma} \max _{s \in[0,1]} \varphi(h(s)) .
$$

If $\varphi$ satisfies the $(P S)$-condition, i.e., a sequence $\left\{u_{n}\right\}$ in $X$ satisfying $\varphi\left(u_{n}\right)$ is bounded and $\varphi^{\prime}\left(u_{n}\right) \rightarrow 0$ as $n \rightarrow \infty$ has a convergent subsequence, then $c$ is a critical value of $\varphi$ and $c>\max \left\{\varphi\left(x_{0}\right), \varphi\left(x_{1}\right)\right\}$. 
Theorem $2.4([8,9])$. Let $X$ be a reflexive real Banach space; let $\Phi, \Psi: X \rightarrow \mathbb{R}$ be two Gâteaux differentiable functionals such that $\Phi$ is sequentially weakly lower semicontinuous, strongly continuous, and coercive and $\Psi$ is sequentially weakly upper semicontinuous. For every $r>\inf _{X} \Phi$, let us put

$$
\varphi(r):=\inf _{u \in \Phi^{-1}((-\infty, r))} \frac{\sup _{v \in \Phi^{-1}((-\infty, r)) \Psi(v)-\Psi(u)}}{r-\Phi(u)}
$$

and

$$
\gamma:=\liminf _{r \rightarrow+\infty} \varphi(r), \quad \delta:=\liminf _{\left.r \rightarrow \inf _{X} \Phi\right)^{+}} \varphi(r)
$$

Then one has

(a) for every $r>\inf _{X} \Phi$ and every $\lambda \in\left(0, \frac{1}{\varphi(r)}\right)$, the restriction of the functional $I_{\lambda}=\Phi-\lambda \Psi$ to $\Phi^{-1}((-\infty, r))$ admits a global minimum, which is a critical point (local minimum) of $I_{\lambda}$ in $X$.

(b) If $\gamma<+\infty$, then for each $\lambda \in\left(0, \frac{1}{\gamma}\right)$, the following alternative holds: either

$\left(b_{1}\right) I_{\lambda}$ has a global minimum; or

$\left(b_{2}\right)$ there exists a sequence $\left\{u_{n}\right\}$ of critical points (local minima) of $I_{\lambda}$ such that $\lim _{n \rightarrow \infty} \Phi\left(u_{n}\right)=+\infty$.

(c) If $\delta<+\infty$, then for each $\lambda \in\left(0, \frac{1}{\delta}\right)$, the following alternative holds: either

$\left(c_{1}\right)$ there exists a global minimum of $\Phi$ which is a local minimum of $I_{\lambda}$; or

$\left(c_{2}\right)$ there exists a sequence of pairwise distinct critical points (local minima) of $I_{\lambda}$, which weakly converges to a global minimum of $\Phi$.

\section{Main results}

Theorem 3.1. Assume that (A) holds and $\left(S_{1}\right)$ there exist constants $c, d>0$ and $\theta \in(0,1)$ such that

$$
f(t, u) \leq c u^{\theta}+d, \quad(t, u) \in(0,1) \times[\epsilon, \infty) .
$$

Then problem (1.1) admits a positive solution in $H$ for $\lambda \geq 1$.

Proof. Clearly, $\Phi$ is continuous and convex, and hence it is weakly sequentially lower semicontinuous. The functional $\Psi$ is well-defined, continuously Gâteaux differentiable and has compact derivative, and hence it is sequentially weakly continuous. Therefore, the functional $J=\Phi-\lambda \Psi$ is weakly sequentially lower semicontinuous.

Next we verify that the functional $J$ is coercive in $H$ for $\lambda \geq 1$.

By (2.4), (2.5) and (3.1), one has

$$
F_{\epsilon}(t, u) \leq \begin{cases}0, & u<\epsilon \\ \frac{c}{2} u^{\theta+1}+d u, & u \geq \epsilon\end{cases}
$$


Therefore,

$$
\begin{aligned}
J(u) & =\frac{1}{2}\|u\|^{2}-\lambda \int_{0}^{1} F_{\epsilon}(t, u) d t \\
& =\frac{1}{2}\|u\|^{2}-\lambda \int_{u<\epsilon} F_{\epsilon}(t, u) d t-\lambda \int_{u \geq \epsilon} F_{\epsilon}(t, u) d t \\
& \geq \frac{1}{2}\|u\|^{2}-\lambda \int_{u \geq \epsilon} F_{\epsilon}(t, u) d t \\
& \geq \frac{1}{2}\|u\|^{2}-\frac{\lambda c}{2}\|u\|^{\theta+1}-\lambda d\|u\| \rightarrow \infty \quad \text { as }\|u\| \rightarrow \infty
\end{aligned}
$$

since $0<\theta<1$. By using [18, Theorem 1.1], we obtain problem (1.1) admits a positive solution in $H$ for $\lambda \geq 1$.

Remark 3.2. Theorem 3.1 gives the existence result of positive solutions for problem (1.1) when the nonlinear term $f$ satisfies sublinear condition at infinity, which is not contained in [5]. Therefore, we extend the results in [5].

Example 1. Consider the following problem

$$
\left\{\begin{array}{l}
-u^{\prime \prime}=\lambda\left(a(t) u^{-\alpha}+u^{\theta}\right), \quad 0<t<1, \\
u(0)=u(1)=0,
\end{array}\right.
$$

where $\lambda \geq 1, a \in L^{1}(0,1), 0<\alpha<1,0<\theta<1$.

Take $\epsilon=1$. Then $(A)$ holds. Moreover, it is easy to verify that the condition $\left(S_{1}\right)$ holds. Therefore, by Theorem 3.1, problem $(S)$ has at least a positive solution for $\lambda \geq 1$.

Theorem 3.3. Assume (A) holds. Moreover, assume that $\left(S_{2}\right)$ There exist two constants $\mu>2$ and $M_{0}>\epsilon$ such that

$$
\mu F_{\epsilon}(t, u) \leq u f(t, u),(t, u) \in(0,1) \times\left[M_{0}, \infty\right) ;
$$

$\left(S_{3}\right)$ There exist constants $\kappa>2, \delta>0$ such that

$$
F_{\epsilon}(t, u) \leq \delta u^{\kappa},(t, u) \in(0,1) \times[\epsilon, \infty) .
$$

Then problem (1.1) with $\lambda=1$ has at least two positive solutions in $H$.

Proof. Step 1. We verify that the functional $\varphi$ satisfies (PS)-condition.

Let a sequence $\left\{u_{n}\right\}$ in $X$ satisfying $J\left(u_{n}\right)$ be bounded and $J^{\prime}\left(u_{n}\right) \rightarrow 0$. By a standard argument, it suffices to show that $\left\{u_{n}\right\}$ is bounded when verifying (PS)-condition.

By (2.4), (2.9) and Lemma 2.1, it is easy to verify that

$$
\begin{aligned}
\left\|u_{n}^{-}\right\|^{2} & =-\left(\int_{0}^{1} f_{\epsilon}\left(t, u_{n}(t)\right) u_{n}^{-}(t) d t+\left\langle J^{\prime}\left(u_{n}\right), u_{n}^{-}\right\rangle\right) \\
& \leq\left\|u_{n}^{-}\right\|_{\infty} \int_{u_{n}<0} f_{\epsilon}\left(t, u_{n}(t)\right) d t+\left\|J^{\prime}\left(u_{n}\right)\right\|\left\|u_{n}^{-}\right\|
\end{aligned}
$$




$$
\begin{aligned}
& \leq\left\|u_{n}^{-}\right\| \int_{u_{n}<0} f_{\epsilon}\left(t, u_{n}(t)\right) d t+\left\|J^{\prime}\left(u_{n}\right)\right\|\left\|u_{n}^{-}\right\| \\
& \leq C\left\|u_{n}^{-}\right\| \int_{0}^{1} \varphi_{\epsilon}(t)^{-\alpha} d t+\left\|J^{\prime}\left(u_{n}\right)\right\|\left\|u_{n}^{-}\right\|,
\end{aligned}
$$

and hence

$$
\left\|u_{n}^{-}\right\|_{\infty} \leq\left\|u_{n}^{-}\right\| \leq C \int_{0}^{1} \varphi_{\epsilon}(t)^{-\alpha} d t+o(1) .
$$

Therefore, $\left\|u_{n}^{-}\right\|_{\infty}$ is bounded.

On the other hand, by $(2.9)$ and $\left(S_{2}\right)$, one has

$$
\begin{aligned}
\left(\frac{\mu}{2}-1\right)\left\|u_{n}\right\|^{2}= & \int_{0}^{1}\left(\mu F_{\epsilon}\left(t, u_{n}\right)-u_{n} f\left(t, u_{n}\right)\right) d t+\mu J\left(u_{n}\right) \\
& -\left\langle J^{\prime}\left(u_{n}\right), u_{n}^{-}\right\rangle \\
\leq & C\left(\int_{u_{n}<\epsilon} \varphi_{\epsilon}(t)^{-\alpha}\left|u_{n}(t)\right| d t+1\right) \\
\leq & C\left(\left\|u_{n}^{-}\right\|_{\infty} \int_{0}^{1} \varphi_{\epsilon}(t)^{-\alpha} d t+1\right) .
\end{aligned}
$$

Combining this with (3.3), we obtain $\left\{u_{n}\right\}$ is bounded in $H$.

Step 2. We apply Theorem 2.2 to show that there exists $M>0$ such that the functional $J$ has a local minimum $u_{0} \in B_{M}=\{u \in H:\|u\| \leq M\}$.

Let $M>0$, which will be determined later. First we claim that $B_{M}$ is bounded and weak sequentially closed. In fact, let $\left\{u_{n}\right\} \subseteq \bar{B}_{M}$ and $u_{n} \rightarrow$ $u$ as $n \rightarrow \infty$. By Mazur Theorem ([18]), there exists a sequence of convex combinations

$$
v_{n}=\sum_{k=1}^{n} \beta_{n_{k}} u_{k}, \quad \sum_{k=1}^{n} \beta_{n_{k}}=1, \quad \beta_{n_{k}} \geq 0, k \in \mathbb{N}
$$

such that $v_{n} \rightarrow u$ in $H$. Since $B_{M}$ is a closed convex set, we have $\left\{v_{n}\right\} \subseteq \bar{B}_{M}$ and $u \in \bar{B}_{M}$. Now we claim that $\varphi$ has a minimum $u_{0} \in \bar{B}_{M}$. It is easy to verify that $\varphi$ is weak sequentially lower semi-continuous on $B_{M}$. Furthermore, $H$ is a reflexive Banach space, so our claim follows from Theorem 2.2.

Without loss of generality, we assume that $J\left(u_{0}\right)=\min _{u \in \bar{B}_{M}} J(u)$. Now we show that

$$
J\left(u_{0}\right)<\inf _{u \in \partial B_{M}} J(u) .
$$

If this is true, then the result of Step 2 holds. In fact, take $M=\epsilon_{1}>0$ satisfying

$$
\frac{1}{2} \epsilon_{1}^{2}-\delta \epsilon_{1}^{\kappa} \geq 0
$$


For any $u \in \partial B_{M},\|u\|=M=\epsilon_{1}$. By (2.8), (3.6), Lemma 2.1 and $\left(S_{3}\right)$, one has

$$
\begin{aligned}
J(u) & =\frac{1}{2} \int_{0}^{1}\left|u^{\prime}\right|^{2} d t-\int_{0}^{1} F_{\epsilon}(t, u) d t \\
& \geq \frac{1}{2} \epsilon_{1}^{2}-\int_{u \geq \epsilon} F_{\epsilon}(t, u) d t \\
& \geq \frac{1}{2} \epsilon_{1}^{2}-\delta \int_{0}^{1}|u|^{\kappa} d t \\
& \geq \frac{1}{2} \epsilon_{1}^{2}-\delta\|u\|_{\infty}^{\kappa} \\
& \geq \frac{1}{2} \epsilon_{1}^{2}-\delta\|u\|^{\kappa}=\frac{1}{2} \epsilon_{1}^{2}-\delta \epsilon_{1}^{\kappa} \geq 0
\end{aligned}
$$

for any $u \in \partial B_{M}$. On the other hand, $J\left(u_{0}\right) \leq J(0) \leq-2 \epsilon^{2}$. So $J\left(u_{0}\right) \leq$ $J(0) \leq-2 \epsilon^{2}<\frac{1}{2} \epsilon_{1}^{2}-\delta \epsilon_{1}^{\kappa} \leq J(u)$ for any $u \in \partial B_{M}$. So (3.5) holds and $u_{0} \in B_{M}$.

Step 3. We show that there exists $u_{1}$ with $\left\|u_{1}\right\|>M$ such that $J\left(u_{1}\right)<$ $\inf _{u \in \partial B_{M}} J(u)$.

Let $\varphi_{1}>0$ be the normalized eigenfunction associated with $\rho_{1}$, where $\rho_{1}$ is the first eigenvalue of

$$
\left\{\begin{array}{l}
-u^{\prime \prime}=\rho u, \quad 0<t<1, \\
u(0)=u(1)=0
\end{array}\right.
$$

By (2.4) and $\left(S_{2}\right)$, one has

$$
F_{\epsilon}(t, u) \geq \begin{cases}-C \varphi_{\epsilon}(t)^{-\alpha}, & 0 \leq u<\epsilon, \\ F_{\epsilon}\left(t, M_{0}\right)\left(\frac{u}{M_{0}}\right)^{\mu}, & u \geq M_{0}\end{cases}
$$

for some $M_{0}>\epsilon$, and it follows that $J\left(R \varphi_{1}\right) \rightarrow-\infty$ as $R \rightarrow \infty$. Therefore, from Step 2 and Step 3, we have $\max \left\{J\left(u_{0}\right), J\left(u_{1}\right)\right\}<\inf _{u \in \partial M} J(u)$. Theorem 2.3 gives the critical point $u^{*}$. Therefore, $u_{0}, u^{*}$ are two critical points of $J$, and hence they are two positive solutions of problem (1.1) with $\lambda=1$.

Remark 3.4. In [5], Agarwal et al. obtained the existence of two positive solutions for problem (1.1) with $\lambda=1$ under the condition $\left(S_{2}\right)$ and the following abstract condition:

$(\mathcal{F})$ There is a constant $M_{1}>0$, independent of $\lambda$, such that $\|u\| \neq M_{1}$ for every solution $u>0$ to

$$
\left\{\begin{array}{l}
-u^{\prime \prime}=\lambda f(t, u), \quad 0<t<1, \\
u(0)=u(1)=0
\end{array}\right.
$$

for each $\lambda \in(0,1]$.

However, in Theorem 3.3, we use the condition $\left(S_{3}\right)$ instead of $(\mathcal{F})$ and obtain the same result. In general, $(\mathcal{F})$ holds if there exists an a priori bound 
of the solutions of the problem, but in most cases, it is not easy to obtain such a priori bound of the solutions. Our condition $\left(S_{3}\right)$ is independent of the bound of the possible solutions and it is clear to be more easily verified.

Theorem 3.5. Suppose (A) holds. Assume that

$$
\begin{aligned}
& \liminf _{\xi \rightarrow+\infty} \frac{2 \int_{0}^{1} \max _{\epsilon \leq u \leq \xi} F_{\epsilon}(t, u) d t+\epsilon C \int_{0}^{1} \varphi_{\epsilon}^{-\alpha}(t) d t}{\xi^{2}} \\
< & \frac{1}{4} \cdot \limsup _{\xi \rightarrow+\infty} \frac{\int_{1 / 4}^{3 / 4} F_{\epsilon}(t, \xi) d t}{\xi^{2}} \leq 1 .
\end{aligned}
$$

Then for every

$$
\lambda \in \Lambda:=\left(\frac{4}{\limsup _{\xi \rightarrow+\infty} \frac{\int_{1 / 4}^{3 / 4} F_{\epsilon}(t, \xi) d t}{\xi^{2}}}, \frac{1}{\liminf _{\xi \rightarrow+\infty} \frac{2 \int_{0}^{1} \max _{\epsilon \leq u \leq \xi} F_{\epsilon}(t, u) d t+\epsilon C \int_{0}^{1} \varphi_{\epsilon}^{-\alpha}(t) d t}{\xi^{2}}}\right),
$$

where $\epsilon, C$ are defined as in $(\mathrm{A})$ and $\varphi_{\epsilon}(t)=\epsilon t(1-t)$, problem (1.1) has an unbounded sequence of positive solutions in $H$.

Proof. We use part (b) of Theorem 2.4 to prove our result. $\Phi$ is continuous and convex, and hence it is weakly sequentially lower semi-continuous. Moreover, $\Phi$ is continuously Gâteaux differentiable and its Gâteaux derivative admits a continuous inverse. The functional $\Psi$ is well-defined, continuously Gâteaux differentiable and has compact derivative, and hence it is sequentially weakly continuous. In particular, one has

and

$$
\Phi^{\prime}(u)(v)=\int_{0}^{1} u^{\prime}(t) v^{\prime}(t) d t
$$

for all $v \in H$.

$$
\Psi^{\prime}(u)(v)=\int_{0}^{1} f_{\epsilon}(t, u(t)) v(t) d t
$$

Next we wish to prove that

$$
\gamma<+\infty
$$

Let $\left\{\xi_{n}\right\}$ be a sequence of positive constants such that for each $n \in \mathbb{N}, \xi_{n}>\epsilon$, $\xi_{n} \rightarrow+\infty$ and

$$
\begin{aligned}
& \lim _{n \rightarrow \infty} \frac{2 \int_{0}^{1} \max _{\epsilon \leq u \leq \xi_{n}} F_{\epsilon}(t, u) d t+\epsilon C \int_{0}^{1} \varphi_{\epsilon}^{-\alpha}(t) d t}{\xi_{n}^{2}} \\
= & \liminf _{\xi \rightarrow+\infty} \frac{2 \int_{0}^{1} \max _{\epsilon \leq u \leq \xi} F_{\epsilon}(t, u) d t+\epsilon C \int_{0}^{1} \varphi_{\epsilon}^{-\alpha}(t) d t}{\xi^{2}} .
\end{aligned}
$$

Take $r_{n}=\frac{1}{2} \xi_{n}^{2}$ for all $n \in \mathbb{N}$. By Lemma 2.1, for all $v \in H$ with $\|v\|^{2}<2 r_{n}$, one has

$$
\max _{t \in[0,1]}|v(t)| \leq \xi_{n} .
$$


Hence by (2.4), one has

$$
\begin{aligned}
\varphi\left(r_{n}\right) & =\inf _{u \in \Phi^{-1}\left(\left(-\infty, r_{n}\right)\right)} \frac{\sup _{v \in \Phi^{-1}\left(\left(-\infty, r_{n}\right)\right)} \Psi(v)-\Psi(u)}{r_{n}-\Phi(u)} \\
& \leq \frac{\sup _{v \in \Phi^{-1}\left(\left(-\infty, r_{n}\right)\right) \Psi(v)-\Psi(0)}}{r_{n}-\Phi(0)} \\
& =\frac{\sup _{v \in \Phi^{-1}\left(\left(-\infty, r_{n}\right)\right)} \Psi(v)+\int_{0}^{1} \int_{0}^{\epsilon} f_{\epsilon}(t, u) d u d t}{r_{n}} \\
& \leq \frac{2 \int_{0}^{1} \max _{\epsilon \leq|u| \leq \xi_{n}} F_{\epsilon}(t, u) d t+\epsilon C \int_{0}^{1} \varphi_{\epsilon}^{-\alpha}(t) d t}{\xi_{n}^{2}} .
\end{aligned}
$$

So

$$
\gamma \leq \liminf _{\xi \rightarrow+\infty} \frac{2 \int_{0}^{1} \max _{\epsilon \leq u \leq \xi} F_{\epsilon}(t, u) d t+\epsilon C \int_{0}^{1} \varphi_{\epsilon}^{-\alpha}(t) d t}{\xi^{2}}<+\infty .
$$

From assumption $\left(S_{4}\right)$ and $(3.8)$, one has

$$
\Lambda \subseteq\left(0, \frac{1}{\gamma}\right)
$$

Fixing $\lambda \in \Lambda,(3.8)$ assures that conclusion (b) of Theorem 2.4 can be used and either $J$ has a global minimum or there exists a sequence $\left\{u_{n}\right\}$ of solutions of problem (1.1) such that $\lim _{n \rightarrow \infty} u_{n}=+\infty$.

Finally, we verify that the functional $J$ has no global minimum in $H$.

By the choice of $\lambda$, we can choose a constant $b$ such that, for each $n \in \mathbb{N}$,

$$
\frac{1}{4} \cdot \sup _{\nu \geq n} \frac{\int_{1 / 4}^{3 / 4} F_{\epsilon}(t, \nu) d t}{\nu^{2}}>b>\frac{1}{\lambda}
$$

and so there exists $\nu_{n} \geq \max \{n, \epsilon\}$ such that

$$
\frac{1}{4} \cdot \frac{\int_{1 / 4}^{3 / 4} F_{\epsilon}\left(t, \nu_{n}\right) d t}{\nu_{n}^{2}}>b .
$$

Consider a function $y_{n} \in H$ defined by

$$
y_{n}(t)=\left\{\begin{array}{l}
4 \nu_{n} t, \quad t \in\left[0, \frac{1}{4}\right] \\
\nu_{n}, \quad t \in\left(\frac{1}{4}, \frac{3}{4}\right] \\
4 \nu_{n}(1-t), \quad t \in\left(\frac{3}{4}, 1\right]
\end{array}\right.
$$

Then

$$
\Phi\left(y_{n}\right)=4 \nu_{n}^{2}
$$


and, using (2.4) and (2.12), we have

$$
\begin{aligned}
J\left(y_{n}\right) & =\Phi\left(y_{n}\right)-\lambda \Psi\left(y_{n}\right)=4 \nu_{n}^{2}-\lambda \int_{0}^{1} F_{\epsilon}\left(t, y_{n}(t)\right) d t \\
& \leq 4 \nu_{n}^{2}-\lambda \int_{1 / 4}^{3 / 4} F_{\epsilon}\left(t, \nu_{n}\right) d t+\frac{\lambda \epsilon^{2}}{2 \nu_{n}} \\
& <4 \nu_{n}^{2}(1-\lambda b)+\frac{\lambda \epsilon^{2}}{2 \nu_{n}} .
\end{aligned}
$$

Combining this with (3.9), we obtain the functional $J$ is unbounded from below and thus has no global minimum.

Therefore, Theorem 2.4 assures that there is a sequence $\left\{u_{n}\right\} \subseteq H$ of critical points of $J$ such that $\lim _{n \rightarrow+\infty}\left\|u_{n}\right\|=+\infty$ and, taking into account the considerations made in Section 2 , the theorem is completely proved.

Remark 3.6. Observe that assumption $\left(S_{4}\right)$ in Theorem 3.5 can be replaced by the more general condition:

$\left(S_{5}\right)$ There exist two sequences $\left\{a_{n}\right\}$ and $\left\{b_{n}\right\}$ with $\epsilon \leq a_{n}<\frac{1}{2 \sqrt{2}} b_{n}$ for every $n \in \mathbb{N}$ and $\lim _{n \rightarrow+\infty} b_{n}=+\infty$ such that

$$
\begin{aligned}
& \lim _{n \rightarrow+\infty} \frac{\int_{0}^{1} \max _{\epsilon \leq u \leq b_{n}} F_{\epsilon}(t, u) d t+\epsilon C \int_{0}^{1} \varphi_{\epsilon}^{-\alpha}(t) d t-\int_{1 / 4}^{3 / 4} F_{\epsilon}\left(t, a_{n}\right) d t+\frac{\epsilon^{3}}{2 a_{n}}}{\frac{b_{n}^{2}}{2}-4 a_{n}^{2}} \\
&<\frac{1}{4} \cdot \limsup _{\xi \rightarrow+\infty} \frac{\int_{1 / 4}^{3 / 4} F_{\epsilon}(t, \xi) d t}{\xi^{2}} \leq 1 .
\end{aligned}
$$

Clearly, by choosing $a_{n}=\epsilon$ for all $n \in \mathbb{N}$, from $\left(S_{5}\right)$ we obtain $\left(S_{4}\right)$. Moreover, if we assume $\left(S_{5}\right)$ instead of $\left(S_{4}\right)$ and take $r_{n}=\frac{1}{2} b_{n}^{2}$ for all $n \in N$, similar to the discussion in Theorem 3.5, we obtain

$$
\begin{aligned}
\varphi\left(r_{n}\right) & =\inf _{u \in \Phi^{-1}\left(\left(-\infty, r_{n}\right)\right)} \frac{\sup _{v \in \Phi^{-1}\left(\left(-\infty, r_{n}\right)\right)} \Psi(v)-\Psi(u)}{r_{n}-\Phi(u)} \\
& \leq \frac{\sup _{v \in \Phi^{-1}\left(\left(-\infty, r_{n}\right)\right)} \Psi(v)-\int_{0}^{1} F_{\epsilon}\left(t, w_{n}(t)\right) d t}{r_{n}-\frac{1}{2}\left\|w_{n}\right\|^{2}} \\
& \leq \frac{\int_{0}^{1} \max _{|u| \leq b_{n}} F_{\epsilon}(t, u) d t-\int_{1 / 4}^{3 / 4} F_{\epsilon}\left(t, a_{n}\right) d t+\frac{\epsilon^{3}}{2 a_{n}}}{\frac{1}{2} b_{n}^{2}-4 a_{n}^{2}} \\
& \leq \frac{\int_{0}^{1} \max _{\epsilon \leq|u| \leq b_{n}} F_{\epsilon}(t, u) d t-\int_{1 / 4}^{3 / 4} F_{\epsilon}\left(t, a_{n}\right) d t+\frac{\epsilon^{3}}{2 a_{n}}}{\frac{1}{2} b_{n}^{2}-4 a_{n}^{2}} \\
& \leq \frac{\int_{0}^{1} \max _{\epsilon \leq u \leq b_{n}} F_{\epsilon}(t, u) d t+\epsilon C \int_{0}^{1} \varphi_{\epsilon}^{-\alpha}(t) d t-\int_{1 / 4}^{3 / 4} F_{\epsilon}\left(t, a_{n}\right) d t+\frac{\epsilon^{3}}{2 a_{n}}}{\frac{b_{n}^{2}}{2}-4 a_{n}^{2}}
\end{aligned}
$$


where

$$
w_{n}(t)=\left\{\begin{array}{l}
4 a_{n} t, \quad t \in\left[0, \frac{1}{4}\right] \\
a_{n}, \quad t \in\left(\frac{1}{4}, \frac{3}{4}\right] \\
4 a_{n}(1-t), \quad t \in\left(\frac{3}{4}, 1\right] .
\end{array}\right.
$$

The conclusion is similar to that of Theorem 3.5 with $\Lambda$ replaced by

$$
\Lambda^{\prime}:=\left(\frac{4}{\limsup _{\xi \rightarrow+\infty} \frac{\int_{1 / 4}^{3 / 4} F_{\epsilon}(t, \xi) d t}{\xi^{2}}}, \frac{1}{\lim _{n \rightarrow+\infty} \frac{\int_{0}^{1} \max _{\epsilon} \leq u \leq b_{n} F_{\epsilon}(t, u) d t+\epsilon C \int_{0}^{1} \varphi_{\epsilon}^{-\alpha}(t) d t-\int_{1 / 4}^{3 / 4} F_{\epsilon}\left(t, a_{n}\right) d t+\frac{\epsilon^{3}}{2 a_{n}}}{\frac{b_{n}^{2}}{2}-4 a_{n}^{2}}}\right) .
$$

Corollary 3.7. Let $f \in \mathbb{C}((0, \infty),[0, \infty))$ satisfy

$$
2 \epsilon \leq f(u) \leq C u^{-\alpha}, \quad u \in(0, \epsilon)
$$

for some $\epsilon, C>0$ and $\alpha \in(0,1)$. Moreover, assume that

$\left(S_{6}\right) \quad \liminf _{\xi \rightarrow+\infty} \frac{2 \int_{\epsilon}^{\xi} f_{\epsilon}(u) d u+\epsilon C \int_{0}^{1} \varphi_{\epsilon}^{-\alpha}(t) d t}{\xi^{2}}<\frac{1}{8} \cdot \limsup _{\xi \rightarrow+\infty} \frac{\int_{\epsilon}^{\xi} f_{\epsilon}(u) d u}{\xi^{2}} \leq 1$.

Then for every

$$
\lambda \in\left(\frac{8}{\limsup _{\xi \rightarrow+\infty} \frac{\int_{\epsilon}^{\xi} f_{\epsilon}(u) d u}{\xi^{2}}}, \frac{1}{\liminf _{\xi \rightarrow+\infty} \frac{2 \int_{\epsilon}^{\xi} f_{\epsilon}(u) d u+\epsilon C \int_{0}^{1} \varphi_{\epsilon}^{-\alpha}(t) d t}{\xi^{2}}}\right),
$$

the problem

$$
\left\{\begin{array}{l}
-u^{\prime \prime}=\lambda f(u), \quad 0<t<1, \\
u(0)=u(1)=0
\end{array}\right.
$$

has an unbounded sequence of positive solutions in $H$.

Corollary 3.8. Let $f \in \mathbb{C}((0, \infty),[0, \infty))$ satisfy $(A)^{\prime}$. Assume that

$$
\liminf _{\xi \rightarrow+\infty} \frac{2 \int_{\epsilon}^{\xi} f_{\epsilon}(u) d u+\epsilon C \int_{0}^{1} \varphi_{\epsilon}^{-\alpha}(t) d t}{\xi^{2}}=0
$$

and

$$
\limsup _{\xi \rightarrow+\infty} \frac{\int_{\epsilon}^{\xi} f_{\epsilon}(u) d u}{\xi^{2}}=8 .
$$

Then for every $\lambda \in(1,+\infty)$, the problem (3.11) has an unbounded sequence of positive solutions in $H$.

Example 2. Let $f:(0, \infty) \rightarrow[0, \infty)$ be the continuous function defined as follows:

$$
f(u)=\left\{\begin{array}{l}
\frac{2}{u^{\alpha}}, \quad u \in(0,1) \\
-2 u+4, \quad u \in[1,2] \\
4 \beta\left[(n+1)^{2}-1\right] \min \{u-n ! n,(n+1) !-u\}, \quad u \in[n ! n,(n+1) !] \\
0, \quad \text { elsewhere }
\end{array}\right.
$$


where $0<\alpha<1,0<\beta \leq 8, n \in \mathbb{N}$ and $n \geq 2$.

Take $\epsilon=1, C=2$. Then $(A)^{\prime}$ holds. Put

$$
a_{n}=n !, \quad b_{n}=n ! n
$$

for each $n \in \mathbb{N}$.

By (2.5), one has

$$
\begin{aligned}
F_{1}\left(a_{n}\right) & =\int_{1}^{a_{n}} f_{1}(u) d u=2 \int_{1}^{2}(-u+2) d u+\sum_{i=1}^{n-1} \int_{b_{i}}^{a_{i+1}} f(u) d u \\
& =1+\sum_{i=1}^{n-1} 4 \beta\left[(i+1)^{2}-1\right]\left[\left(a_{i+1}-b_{i}\right)\left(\frac{a_{i+1}+b_{i}}{2}-(i !) i\right) \frac{1}{2}\right] \\
& =1+\sum_{i=1}^{n-1} \beta\left[(i+1)^{2}-1\right](i !)^{2}=1+\beta\left[(n !)^{2}-1\right]
\end{aligned}
$$

for each $n \in \mathbb{N}$. Therefore, we obtain

$$
\lim _{n \rightarrow+\infty} \frac{F_{1}\left(a_{n}\right)}{a_{n}^{2}}=\beta
$$

and by simple calculations, one has

$$
\limsup _{\xi \rightarrow+\infty} \frac{F_{1}(\xi)}{\xi^{2}}=\limsup _{\xi \rightarrow+\infty} \frac{\int_{1}^{\xi} f_{1}(u) d u}{\xi^{2}}=\beta
$$

On the other hand, it is easy to verify that

$$
F_{1}\left(b_{n}\right)=\int_{1}^{b_{n}} f_{1}(u) d u=1+\beta\left[(n !)^{2}-1\right] .
$$

One has

$$
\lim _{n \rightarrow+\infty} \frac{2 F_{1}\left(b_{n}\right)+2 \int_{0}^{1} \varphi_{1}^{-\alpha}(t) d t}{b_{n}^{2}}=0
$$

for which

$$
\liminf _{\xi \rightarrow+\infty} \frac{2 F_{1}(\xi)+2 \int_{0}^{1} \varphi_{1}^{-\alpha}(t) d t}{\xi^{2}}=\liminf _{\xi \rightarrow+\infty} \frac{2 \int_{1}^{\xi} f_{1}(u) d u+2 \int_{0}^{1} \varphi_{1}^{-\alpha}(t) d t}{\xi^{2}}=0 .
$$

Therefore,

$$
0=\liminf _{\xi \rightarrow+\infty} \frac{2 \int_{1}^{\xi} f_{\epsilon}(u) d u+2 \int_{0}^{1} \varphi_{1}^{-\alpha}(t) d t}{\xi^{2}}<\frac{\beta}{8}=\frac{1}{8} \cdot \limsup _{\xi \rightarrow+\infty} \frac{\int_{1}^{\xi} f_{\epsilon}(u) d u}{\xi^{2}} \leq 1 .
$$

Hence, according to Corollary 3.6, for each $\lambda>\frac{8}{\beta}$, the problem

$$
\left\{\begin{array}{l}
-u^{\prime \prime}=\lambda f(u), \quad 0<t<1 \\
u(0)=u(1)=0
\end{array}\right.
$$

has an unbounded sequence of pairwise distinct positive solutions. 


\section{References}

[1] R. P. Agarwal and D. O'Regan, Singular Differential and Integral Equations with Applications, Kluwer Academic Publishers, Dordrecht, 2003.

[2] Singular boundary value problems for superlinear second order ordinary and delay differential equations, J. Differential Equations 130 (1996), no. 2, 333-355.

[3] _ Existence theory for single and multiple solutions to singular positone boundary value problems, J. Differential Equations 175 (2001), no. 2, 393-414.

[4] — Existence criteria for singular boundary value problems with sign changing nonlinearities, J. Differential Equations 183 (2002), no. 2, 409-433.

[5] R. P. Agarwal, K. Perera, and D. O'Regan, Multiple positive solutions of singular problems by variational methods, Proc. Amer. Math. Soc. 134 (2006), no. 3, 817-824.

[6] R. Aris, The Mathematical Theory of Diffusion and Reaction in Permeable Catalysts, Clarendon Press, 8, Oxford, 1975.

[7] J. V. Baxley, A singular nonlinear boundary value problem: membrane response of a spherical cap, SIAM J. Appl. Math. 48 (1988), no. 3, 497-505.

[8] G. Bonanno and G. Molica Bisci, Infinitely many solutions for a boundary value problem with discontinuous nonlinearities, Bound. Value Probl. 2009 (2009), Article ID 670675, 20 pages.

[9] G. Bonanno and B. Di Bella, Infinitely many solutions for a fourth-order elastic beam equation, Nonlinear Differential Equations Appl. 18 (2011), no. 3, 357-368.

[10] A. Callegari and A. Nachman, Some singular nonlinear differential equations arising in boundary layer theory, J. Math. Anal. Appl. 64 (1978), no. 1, 96-105.

[11] J. Chu and D. O'Regan, Multiplicity results for second order non-autonomous singular Dirichlet systems, Acta Appl. Math. 105 (2009), no. 3, 323-338.

[12] J. Á. Cid, Ó. L. Pouso, and R. L. Pouso, Existence of infinitely many solutions for second-order singular initial value problems with an application to nonlinear massive gravity, Nonlinear Anal. Real World Appl. 12 (2011), no. 5, 2596-2606.

[13] L. Erbe and R. Mathsen, Positive solutions for singular nonlinear boundary value problems, Nonlinear Anal. 46 (2001), no. 7, 979-986.

[14] P. Habets and F. Zanolin, Upper and lower solutions for a generalized Emden-Fowler equation, J. Math. Anal. Appl. 181 (1994), no. 3, 684-700.

[15] X. He and W. Zou, Infinitely many solutions for a singular elliptic equation involving critical sobolev-Hardy exponents in $\mathbb{R}^{N}$, Acta Math. Sci. Ser. B Engl. Ed. 30 (2010), no. $3,830-840$.

[16] K. Lan, Multiple positive solutions of semilinear differential equations with singularities, J. London Math. Soc. (2) 63 (2001), no. 3, 690-704.

[17] K. Lan and J. R. Webb, Positive solutions of semilinear differential equations with singularities, J. Differential Equations 148 (1998), no. 2, 407-421.

[18] J. Mawhin and M. Willem, Critical Point Theory and Hamiltonian Systems, Springer, 1989.

[19] A. Nachman and A. Callegari, A nonlinear singular boundary value problem in the theory of pseudoplastic fluids, SIAM J. Appl. Math. 38 (1980), no. 2, 275-282.

[20] S. Taliaferro, A nonlinear singular boundary value problem, Nonlinear Anal. 3 (1979), no. 6, 897-904.

[21] E. Zeidler, Nonlinear Functional Analysis and its Applications. III, Springer-Verlag, 1985

Juntao Sun

SCHOOL OF SCIENCE

Shandong University of TeChnology

Zibo, 255049 Shandong, P. R. China

E-mail address: sunjuntao2008@163.com 
JiFENG CHU

Department of Mathematics

College of Science

Hohai University

NAnjing, 210098 Jiangsu, P. R. China

E-mail address: jifengchu@126.com 